\title{
Facteurs de risque de la qualité bactériologique du lait de traite et de l'infection mammaire chez la vache laitière
}

\author{
B Faye, F Lescourret, N Dorr \\ INRA laboratoire d'écopathologie, Theix, 63122 St-Genès-Champanelle, France
}

La définition de la qualité bactériologique du lait peut s'appuyer sur plusieurs critères tels que la présence de germes pathogènes dans le lait de traite (infection intramammaire), la survenue d'une infection cliniquement observable (mammite clinique) ou dépistable par le comptage des cellules somatiques (mammite subclinique), la gravité des signes cliniques. De nombreux facteurs agissent sur la santé de la mamelle et la qualité hygiénique du lait par de multiples voies, aboutissant ainsi un modèle complexe.

L'étude présentée ici est le résultat d'un suivi écopathologique réalisé pendant 4 ans dans 47 élevages laitiers intensifiés de Bretagne (France). 4129 vaches pie-noires ont fait l'objet d'un prélèvement de lait en début de lactation (soit au total 7852 analyses). Six critères définissant un complexe "atteinte de la mamelle" représentaient les variables à expliquer : taux de prévalence de germes pathogènes majeurs, mineurs ou rares, taux d'incidence des mammites cliniques en début de lactation, taux de prévalence des mammites subcliniques lors des deux premiers contrôles laitiers, taux de gravité clinique (Faye et al, 1994, Vet Res, 25, 213-218). Trente-quatre variables potentiellement explicatives (bâtiment et hygiène générale, pratiques de traite et de tarissement, caractéristiques de la salle de traite, niveau de production, facteurs alimentaires, maladies extra-mammaires) ont été testées.

Le traitement statistique s'est appuyé sur l'analyse factorielle des correspondances avec variables instrumentales ou analyse canonique des correspondances (Chessel et al, 1987, Rev Stat Appl, 35, 55-72). Cette méthode représente une généralisation de la régression appliquée à deux tableaux de données, l'un étant explicatif de l'autre.

Le résultat de l'analyse a indiqué que la part de la variance du tableau "complexe de l'atteinte mammaire", expliquée par le tableau "facteurs de risque potentiels", était de $50 \%$ soit un coefficient de corrélation de 0,70 . Les combinaisons de variables les plus contributives étaient les suivantes: logettes en bâtiment fermé -absence d'épandage de superphosphates dans la litière -absence de trempage et de lavettes individuelles -faible note d'état corporel au vêlage pour expliquer les taux élevés d'infections intramammaires (IMI) avec des germes pathogènes mineurs; faible note d'état corporel au vêlage -faible fréquence de distribution de vitamines A, D3, E -litière humide -production laitière élevée pour expliquer les taux élevés d'IMI avec des germes majeurs ; stabulation libre paillée distribution de ray-grass anglais pour expliquer les taux élevés de mammites cliniques; stabulation libre paillée -épandage de superphosphates -lavette collective -absence de distribution de vitamines pour expliquer les taux élevés de mammites subcliniques.

Les plans de prévention des mammites s'intéressent préférentiellement aux pratiques de traite et à la maintenance de la machine à traire. Plusieurs résultats semblent indiquer que ces dernières représentent aujourd'hui plus des indicateurs de la présence de troubles mammaires dans l'exploitation que de réels facteurs de risque. A l'inverse, les pratiques alimentaires sont rarement prises en compte dans la littérature. Nous pensons que des progrès non négligeables peuvent être obtenus en incluant ces pratiques dans les études sur les facteurs de risque des mammites. 